\title{
Estudio comparativo de la percepción de la violencia familiar entre adolescentes, usuarios y no usuarios de drogas ilícitas
}

\author{
Jorge Luis Arellanez Hernández', Arminda Tlaxcalteco González', Daniela Morales Hernández' \\ I Instituto de Investigaciones Psicológicas, Universidad Veracruzana, México
}

\section{RESUMEN}

Introducción: La violencia en México se ha incrementado de manera sustantiva en lo que va del presente siglo, y el grupo familiar no ha quedado exento de este tipo de eventos, afectando principalmente a los adolescentes. Veracruz es uno de los estados en los que se ha registrado un incremento sustantivo de este tipo de violencia en el grupo familiar. Objetivo: Con el objetivo de explorar en estudiantes de telebachillerato usuarios y no usuarios de drogas ilícitas su percepción acerca de la violencia familiar, e identificar su relación con el uso de drogas. Método: se llevó a cabo un estudio transversal, correlacional, comparativo, en el que participaron 667 estudiantes del municipio de Alto Lucero de Gutiérrez Barrios, Veracruz, México. Resultados: $13.2 \%$ había usado alguna droga ilícita al menos una vez, $86.8 \%$ no había consumido. El grupo de usuarios de drogas ilícitas percibe en una proporción significativamente mayor eventos violentos entre hermanos, de padres a hijos, entre padres, y de hijos a padres. La violencia ejercida fue predominantemente física y psicológica. Discusión y conclusiones: La violencia entre hermanos resultó ser predictor del consumo de drogas ilícitas; lo que permite concluir que es un tema por considerar en la atención del grupo familiar en particular cuando hay hijos en la etapa de la adolescencia.

\begin{abstract}
Introduction: Violence in Mexico has increased so far this century, the family has been affected by this type of events, the population group most affected is that of adolescents. Veracruz is one of the states that has registered an increase in family violence. Aim: explore in high school student's (users and non-users of illicit drugs) perception of family violence, as well as to identify the relationship of this violence with the use of drugs. Method: a cross-sectional, correlational, comparative study was designed. 667 students from Alto Lucero de Gutiérrez Barrios, Veracruz, Mexico. Results: $13.2 \%$ users of drugs at some time in life, $86.8 \%$ non-users. Drug users perceive a significantly higher proportion of violent events between siblings, from parents to children, between parents, and from children to parents. Discussion and conclusions: The violence exercised was predominantly physical and psychological. Sibling violence turned out to be a predictor of illicit drug use. This is a topic to analyze especially in groups of families that have at least one adolescent child.
\end{abstract}

Keywords: family violence, psychological violence, physical violence, drug use, adolescents.

Palabras clave: violencia familiar, violencia psicológica, violencia física, uso de drogas, adolescentes.

\section{Autor de correspondencia:}

Dr. Jorge Luis Arellanez Hernández. Instituto de Investigaciones Psicológicas, Universidad Veracruzana. Av. Dr. Luis Castelazo Ayala s/n, col. Industrial Ánimas, C.P. 91190, Xalapa, Veracruz. Tel. +52 (228) 84189 13, fax +52 (228) 84189 14. Correo

electrónico: jarellanez@uv.mx

Recibido: 26 de julio de 2018

Aceptado: 23 de octubre de 2018

doi: 10.28931/riiad.2018.2.02 


\section{INTRODUCCIÓN}

Uno de los fenómenos sociales que ha irrumpido en el mundo en el presente siglo y que ha trastocado la vida cotidiana de millones de personas es la violencia, expresada a través de múltiples manifestaciones. Tal ha sido su magnitud e impacto que en el año 2003 la Organización Mundial de la Salud (OMS) lo consideró como un problema de salud pública, clasificando sus distintas expresiones en: autoinfligida, interpersonal y colectiva (Krug, Dahlberg, Mercy, Zwi, \& Lozano, 2003).

En el presente manuscrito se consideró como objeto de estudio la violencia interpersonal, particularmente la que se presenta en el contexto familiar. La violencia familiar, al igual que la violencia general, no es un tema nuevo; pero fue hasta la década de los 60 que se visualizó como un problema grave, sobre todo por el maltrato cada vez más frecuente que se observaba hacia los niños, las niñas y los adolescentes. Para la década de 1970 el movimiento feminista puso en evidencia una alta ocurrencia de eventos violentos contra las mujeres en el contexto familiar. Para fines del siglo XX se hizo evidente que la presencia de eventos violentos en el grupo familiar era más común de lo que se creía (Corsi, 1994).

En 1999, el Instituto Nacional de Estadística y Geografía (INEGI) llevó a cabo una Encuesta sobre Violencia Intrafamiliar en la Zona Metropolitana de la Ciudad de México, encontrando que los actos violentos se presentaban sobre todo en hogares dirigidos por hombres (32.5\%), en comparación con los dirigidos por mujeres (22.0\%). También identificaron que quienes agredían con mayor frecuencia eran: jefe del hogar (49.5\%) y cónyuge $(44.1 \%)$; las víctimas más comúnmente afectadas fueron: hijas e hijos (44.9\%) y cónyuge (38.9\%). Las formas más frecuentes de violencia física fueron los golpes con el puño (42.0\%), bofetadas (40.0\%) y golpes con objetos (23.0\%), mientras que la expresión de maltrato emocional más común implicó gritos (86.0\%; INEGI, 2003).

Esta situación coadyuvó a que el gobierno federal mexicano estableciera algunos criterios para la Atención Médica de la Violencia Familiar, a través de la Norma Oficial Mexicana 046 (NOM-046-SSA2-2005), definiéndola como el "acto u omisión, único o repetitivo, cometido por un miembro de la familia en contra de otro $u$ otros integrantes de la misma, sin importar si la relación se da por parentesco sanguíneo, de afinidad, o civil mediante matrimonio, concubinato $u$ otras relaciones de hecho, independientemente del espacio físico donde ocurra. La violencia familiar comprende: abandono, maltrato físico, maltrato psicológico, maltrato sexual, maltrato económico y violencia sexual" (Secretaría de Salud, 2009).

En años recientes, el INEGI ha realizado la Encuesta Nacional sobre la Dinámica de las Relaciones en los
Hogares (ENDIREH). En su versión 2016 reportó que, en los 12 meses previos a la encuesta, $10.3 \%$ de los participantes fue víctima de algún acto violento por parte de algún integrante de su familia. $8.1 \%$ de las mujeres experimentó violencia emocional. Los agresores más señalados fueron los hermanos, el padre y la madre; sin embargo, los principales agresores sexuales fueron tíos y primos. Las agresiones más ocurridas en el hogar fueron emocionales $(59.6 \%)$, físicas $(16.9 \%)$ y de tipo económico-patrimonial (17.5\%); en menor porcentaje, las de tipo sexual (6.0\%; INEGI, 2017).

El estado de Veracruz, escenario donde se llevó a cabo el presente estudio, registró eventos de violencia familiar por encima del promedio nacional en ese periodo $(11.1 \%)$, sobre todo la de carácter emocional y económico-patrimonial (INEGI, 2017). Durante el segundo semestre del 2016 en el municipio de Alto Lucero de Gutiérrez Barrios, localizado en la zona centro del estado de Veracruz, se presentaron situaciones de violencia que alteraron de manera sustantiva la vida familiar de sus habitantes (León Carmona, 2016).

De acuerdo con la literatura internacional, la diversidad de expresiones violentas en el grupo familiar está impactando en mayor medida a los hijos e hijas. Esta situación puede colocarlos en una condición de vulnerabilidad, propiciando desajustes en su comportamiento y estabilidad emocional (Gallegos-Guajardo, Ruvalcaba-Romero, Castillo-López, \& Ayala-Díaz, 2016). Por ejemplo, un estudio realizado con 35 niños y adolescentes cubanos que asistieron a consulta de Psiquiatría Infanto-Juvenil y Psicología, a los que les fue diagnosticado algún trastorno psicológico (trastorno de adaptación, trastorno por déficit de atención e hiperactividad y fobia específica, entre otros), se encontró que $82.8 \%$ tenía antecedentes de violencia familiar; de éstos, $100 \%$ señaló haber padecido violencia psicológica y $59 \%$, violencia física (Martínez Almodóvar, López Triana, Díaz Montesinos, \& Teseiro Plá, 2015).

En otro estudio realizado con 304 estudiantes colombianos de 10 a 18 años, se encontró una alta correlación entre la ausencia de vinculación afectiva y apoyo entre la familia y la emisión de conductas de enfrentamiento entre estudiantes (bullying, ya sea como víctimas y/o agresores); en particular, la investigación puso en evidencia la relación entre estudiantes agresores con la percepción del funcionamiento de su familia, moderadamente o gravemente disfuncional (Uribe, Orcasita, \& Aguillón, 2012). Así también, Cabrera-Fuentes (2011), en una muestra de 123 estudiantes de Ecuador, encontró una relación significativa que sugiere que a menor nivel de funcionalidad familiar, mayor nivel de bullying.

Por su parte, un estudio realizado con 133 estudiantes de bachillerato mexicanos de 15 a 19 años, cuyo 
objetivo fue estudiar la relación entre el funcionamiento familiar y la exposición a la violencia, se encontró que los hombres están más expuestos a situaciones de violencia en la calle y la escuela; también reportaron mayor flexibilidad, cohesión, satisfacción y comunicación familiar (Gallegos-Guajardo, Ruvalcaba-Romero, Castillo-López, \& Ayala-Díaz, 2016).

Si bien es de reconocerse que la violencia en el contexto familiar ha sido ampliamente estudiada, lo cierto es que hasta la presente década se ha empezado a estudiar la violencia que se llega a ejercer de los hijos hacia los padres. Por ejemplo, en un estudio con 1,427 adolescentes españoles (728 mujeres, 682 varones), los resultados mostraron que en $65.8 \%$ de los casos las agresiones verbales menos severas son altamente frecuentes, como hacer llorar a los padres, mientras que las agresiones físicas son mucho menos frecuentes (7.2\%). La violencia de los hijos hacia los padres se asoció con la exposición a la violencia familiar en general, a una baja disciplina ejercida por los padres, amigos con problemas de conducta, síntomas de depresión, conducta delincuente, consumo de drogas, impulsividad, creencias justificadoras de la violencia y de grandiosidad. Se identificó que los varones ejercen más la violencia física, mientras que las mujeres ejercen más actos de violencia verbal. Las madres sufren más actos de violencia verbal que los padres (Calvete, Orue, \& Sampedro, 2011). Otro estudio más reciente, también en población española, evaluó a 1,698 adolescentes ( 870 hombres y 828 mujeres) con edades entre 12 y 17 años, con respecto a la presencia de violencia filial-parental, encontrando que los bajos niveles de afecto y comunicación se asociaban a todas las formas de este tipo de violencia de los hijos hacia ambos padres (Calvete, Gámez-Guadix, \& Orue, 2014).

Otros estudios reportan que haber sido víctima de algún evento violento en el contexto familiar puede colocar a la persona en riesgo para consumir alguna sustancia psicoactiva, como una estrategia para afrontar ese malestar afectivo (Caballero \& Ramos, 2004; Centros de Integración Juvenil, 2007); como una forma de atenuar el deterioro de las relaciones familiares (Butters, 2002), la ruptura o el conflicto en las relaciones entre padres e hijos (El-Sheikh \& Flanagan, 2001; Harrington \& Metzler, 1997; Ledoux, Miller, Choquet \& Plant, 2002); asimismo, se ha encontrado que las familias con mayores niveles de desorganización (caracterizadas por enfermedad mental de los padres, consumo de sustancias de los padres y/o familias no intactas) tienen más probabilidades de tener hijos consumidores de drogas, tanto legales como ilegales (Becoña et al., 2012).

Con base en estos elementos, surgió el interés por explorar en estudiantes de Telebachillerato del muni- cipio de Alto Lucero de Gutiérrez Barrios, Veracruz, su percepción acerca de la violencia familiar e identificar si esta condición puede estar asociada al uso de drogas ilícitas.

\section{MÉTODO}

\section{Diseño}

Para lograr el objetivo se diseñó un estudio transversal, correlacional con dos grupos de comparación: Usuarios y No usuarios de drogas ilícitas.

\section{Participantes}

En el estudio participaron estudiantes de Telebachillerato del municipio de Alto Lucero, Veracruz, México, modalidad educativa que predominantemente se encuentra en contextos rurales o semi-rurales y que en general ha sido poco estudiada. La mayoría de los estudios realizados con estudiantes de enseñanza media involucra estudiantes con modalidades educativas tradicionales, es decir, del sistema escolarizado que sobre todo se localiza en zonas urbanas. Asimismo, como se ha señalado, en los últimos tres años se han presentado en el municipio altos índices de violencia interpersonal que han alterado de manera sustantiva la vida familiar de sus habitantes (León Carmona, 2016).

A través de un muestreo no probabilístico, por conveniencia, se invitó a participar en el estudio a todos los estudiantes de los cinco planteles de Telebachillerato localizados en el municipio (Dirección General de Telebachillerato, 2017); como único criterio de inclusión se planteó que fueran alumnos regulares inscritos en el periodo 2016-2017, con una participación voluntaria. Se encuestó a 667 estudiantes. Hubo una participación ligeramente mayor de mujeres (52.0\%) que de varones (46.5\%; 1.5\% no proporcionó información), con una edad promedio de 16.5 años $(D E=1.14)$, en un rango de 15 a 22 años. 34.9\% estaba en primer año, 33.0\% en segundo y $30.1 \%$ en tercero (1.9\% no dio información). $73.5 \%$ sólo se dedicaba a estudiar y $23.7 \%$ realizaba además alguna actividad laboral (2.8\% no contestó).

\section{Instrumento}

Se diseñó un cuestionario exprofeso, en el que además de explorar algunas características sociodemográficas, incluía:

- Escala percepción de la violencia familiar. Instrumento creado y validado con adolescentes mexicanos que consta de 19 situaciones que pueden presen- 
tarse entre cónyuges, de padres a hijos, de hijos a padres, así como entre hermanos (seis exploran violencia psicológica, ocho violencia física y cinco violencia sexual, lo que hace un total de 67 reactivos), con cinco opciones de respuesta tipo Likert: 0 , nunca ha pasado; 1, casi nunca; 2, a veces; 3, casi siempre; y 4, siempre me ha pasado (Tlaxcalteco González, Arellanez Hernández, \& Márquez Barradas, 2017). En el presente estudio se realizó un análisis para corroborar su calidad psicométrica, en el que se obtuvo un coeficiente de confiabilidad global y por subescala por encima de .90 y una validez (a través de un análisis factorial) que arrojó tres factores que coinciden con la original, que explicaron una varianza superior al $40 \%$.

- Cuadro de preguntas que exploran el patrón de consumo de drogas. Este bloque explora de forma descriptiva el uso de alcohol, tabaco y otras drogas (mariguana, solventes inhalables, cocaína, heroína, anfetaminas, éxtasis y tranquilizantes), tanto alguna vez en la vida, como en el último año y en el último mes.

\section{Procedimiento}

En coordinación con las autoridades de Zona de Supervisión Xalapa "A" de Telebachillerato y de los planteles participantes (Dirección General de Telebachillerato, 2017), se planeó la aplicación del instrumento durante marzo y abril del 2017. El equipo de investigadores capacitó a un grupo de orientadores y profesores de las escuelas para coordinar la captación de alumnos.

\section{Consideraciones éticas}

En cada plantel se invitó a participar a todos los estudiantes y se solicitó su autorización a través de su consentimiento verbal, de acuerdo con los principios científicos y éticos considerados en la Declaración de Helsinki (Asociación Médica Mundial, 2013), la Ley General de Salud (DOF, 2017) y el Reglamento de la Ley General de Salud en Materia de Investigación para la Salud (DOF, 2014), cuidando en todo momento el respeto a la dignidad de los participantes, la confidencialidad en el manejo de la información y el anonimato de sus respuestas.

Las autoridades de la escuela dieron su consentimiento verbal para llevar a cabo el levantamiento de información, y frente a grupo, a cada alumno se le explicó que su participación consistiría en contestar un grupo de preguntas no invasivas con un riesgo mínimo para alterar su salud mental o física, así como el manejo ético que habría de sus respuestas. Quienes dieron su asentimiento verbal para participar fueron reunidos en grupos según las actividades académicas.

\section{Plan de análisis}

De manera inicial se realizó un análisis descriptivo para explorar el consumo de sustancias psicoactivas y, con base en ello, se estructuraron dos grupos: el de No usuarios y el de Usuarios de drogas ilícitas. Posteriormente, se analizó la normalidad de los datos a través de la prueba Kolmogorov-Smirnov. A través de estadística inferencial, se realizaron pruebas de Chi Cuadrada, $t$ de Student para muestras independientes, según fuese el caso, para identificar posibles diferencias entre los dos grupos de estudio. Asimismo, a través de la prueba $d$ de Cohen se exploró la magnitud del efecto de las variables de la violencia familiar. Finalmente, se elaboró un análisis de regresión logística para identificar posibles factores predictores del consumo, se consideraron como variables independientes la percepción de violencia conyugal, de padres a hijos, de hijos a padres y entre hermanos; y como variable dependiente el consumo o no de drogas ilícitas alguna vez. En las variables del modelo se eliminaron los valores extremos y se crearon variables dummy para lograr una distribución relativamente simétrica. Así fue como se sentaron las bases para cubrir los criterios mínimos y elaborar un análisis de regresión logística a través del método Enter, que permite conducir el análisis en función de los resultados que va obteniendo y decidir qué variables se introducen o extraen del modelo.

\section{RESULTADOS}

En primera instancia se exploró el consumo de sustancias psicoactivas en los estudiantes; el alcohol fue la sustancia con mayores porcentajes de consumo tanto alguna vez, como en el último año y en el último mes. De los 667 estudiantes participantes en el estudio, cerca de la mitad reportó haber consumido al menos una vez una bebida con alcohol, una cuarta parte lo hizo en el último año y poco más del 15\% dijo haber consumido al menos una bebida alcohólica en los últimos 30 días. La segunda sustancia que reportó un alto porcentaje de consumo fue el tabaco; una cuarta parte de los estudiantes reconoció haber fumado al menos en una ocasión un cigarrillo de esta sustancia; alrededor del 15\% fumó en el último año y cerca del 10\% reconoció haber fumado en el mes previo al levantamiento de la encuesta (Tabla 1).

La droga ilícita de mayor porcentaje de consumo tanto alguna vez en la vida, como en el último año y en el último mes fue la mariguana. Las sustancias que ocuparon el segundo lugar fueron los tranquilizantes, tanto alguna vez en la vida como en el último año; la cocaína fue la segunda droga de consumo en el último mes. La tercera 
Tabla 1

Frecuencia y porcentajes del consumo de sustancias psicoactivas $(n=667)$

\begin{tabular}{|c|c|c|c|c|c|c|}
\hline \multirow[t]{2}{*}{ Sustancia } & \multicolumn{2}{|c|}{ Alguna vez } & \multicolumn{2}{|c|}{ Último año } & \multicolumn{2}{|c|}{ Último mes } \\
\hline & Frecuencia & $\%$ & Frecuencia & $\%$ & Frecuencia & $\%$ \\
\hline Tabaco & 168 & 25.2 & 95 & 14.2 & 64 & 9.6 \\
\hline Alcohol & 304 & 45.6 & 165 & 24.7 & 104 & 15.6 \\
\hline Drogas ilícitas & 88 & 13.2 & 43 & 6.4 & 33 & 4.9 \\
\hline Mariguana & 45 & 6.7 & 22 & 3.3 & 16 & 2.4 \\
\hline Solventes inhalables & 7 & 1.0 & 5 & 0.7 & 4 & 0.6 \\
\hline Cocaína & 13 & 1.9 & 8 & 1.2 & 8 & 1.2 \\
\hline Heroína & 6 & 0.9 & 4 & 0.6 & 3 & 0.4 \\
\hline Anfetaminas & 5 & 0.7 & 2 & 0.3 & 3 & 0.4 \\
\hline Éxtasis & 10 & 1.5 & 4 & 0.6 & 3 & 0.4 \\
\hline Tranquilizantes & 26 & 3.9 & 13 & 1.9 & 7 & 1.0 \\
\hline
\end{tabular}

droga de mayor consumo alguna vez y en el último año fue la cocaína, mientras que los tranquilizantes ocuparon este lugar durante el último mes (Tabla 1).

De acuerdo con los objetivos planteados en el estudio, se identificó que 579 estudiantes reportaron no haber usado alguna droga ilícita y conformaron el grupo denominado No usuarios (86.8\%); mientras que 88 jóvenes reconocieron haber consumido alguna vez una droga ilícita y fueron quienes constituyeron el grupo de Usuarios de drogas ilícitas (13.2\%).

\section{Comparación de características sociodemográficas por grupo de estudio}

Tanto en el grupo de No usuarios como en el de Usuarios de drogas ilícitas hubo una participación ligeramente mayor de mujeres (Tabla 2). Ha de recordarse que en general participó una mayor proporción de mujeres. Las diferencias entre los grupos no resultaron estadísticamente significativas, $X^{2}(1)=0.202 ; p=.653$.

La edad promedio de los estudiantes del grupo de No usuarios fue ligeramente menor, de 16.4 años ( $D E=1.14$ ), la del grupo de Usuarios de drogas ilícitas fue de 16.7 años en promedio ( $D E=1.12)$, no mostrando diferencias significativas, $t$ de Student $(662)=1.797 ; p=.073$.

En el grupo de Usuarios de drogas ilícitas hubo un porcentaje de alumnos de tercer grado ligeramente mayor, en comparación con los No usuarios; de hecho, en estos últimos hay un porcentaje de adolescentes de primer año ligeramente más alto; las diferencias observadas no fueron estadísticamente significativas, $X^{2}(2)=1.380$; $p=.502$. Si bien la mayoría de los estudiantes se dedicaba sólo a estudiar, una mayor proporción de alumnos del grupo de Usuarios de drogas ilícitas realizaba ade-
Tabla 2

Porcentajes de características sociodemográficas por grupo de estudio $(N=667)$

\begin{tabular}{lcc}
\hline & $\begin{array}{c}\text { No usuarios } \\
(\mathrm{n}=579)\end{array}$ & $\begin{array}{c}\text { Usuarios de } \\
\text { drogas ilícitas } \\
(\mathrm{n}=88)\end{array}$ \\
\hline $\begin{array}{l}\text { Sexo } \\
\text { Hombres }\end{array}$ & 46.8 & 49.4 \\
Mujeres & 53.2 & 50.6 \\
Grado escolar & & \\
Primero & 36.3 & 31.0 \\
Segundo & 33.7 & 33.3 \\
$\quad$ Tercero & 30.0 & 35.6 \\
Ocupación & & \\
Sólo estudia & 76.2 & 71.4 \\
Estudia y trabaja & 23.8 & 28.6 \\
\hline
\end{tabular}

más alguna actividad laboral. Al comparar la ocupación entre los No usuarios y los Usuarios de drogas ilícitas no se encontraron diferencias significativas, $X^{2}(1)=0.918$; $p=0.338$ (Tabla 2).

\section{Percepción de violencia familiar por grupo de estudio}

En general, los estudiantes participantes percibieron una baja frecuencia de situaciones relacionadas con la violencia familiar; en un rango de cero a cuatro puntos, la calificación promedio osciló entre 1.00 y 1.18, la violencia entre hermanos fue la más frecuente y la que menos se presentó fue la de hijos hacia padres (Tabla 3). Empero, 
a pesar de registrar una frecuencia relativamente baja en expresiones de violencia familiar, los estudiantes del grupo de Usuarios de drogas ilícitas presentaron en promedio una presencia significativamente mayor de situaciones violentas entre hermanos, $t$ de Student (637) $=$ 4.684, $p=.000$, de padres e hijos, $t$ de Student (94.377) $=3.954, p=.000$, entre los padres (violencia conyu- gal), $t$ de Student (95.072) = 3.044, $p=.003)$, así como de hijos a padres, $t$ de Student $(96.752)=4.052, p=$ .000).

El tamaño del efecto observado en todas las expresiones de violencia que se presentan en el grupo familiar es grande, ya que el valor de la prueba d de Cohen es superior a .50 .

Tabla 3

Puntuaciones de violencia familiar por grupo de estudio $(N=667)$

\begin{tabular}{|c|c|c|c|c|c|c|c|}
\hline & \multicolumn{3}{|c|}{$\begin{array}{c}\text { No usuarios } \\
(n=579)\end{array}$} & \multicolumn{3}{|c|}{$\begin{array}{l}\text { Usuarios de drogas ilícitas } \\
\qquad(n=88)\end{array}$} & \multirow{2}{*}{$\begin{array}{c}\text { Tamaño del } \\
\text { efecto } \\
d \text { de Cohen }\end{array}$} \\
\hline & $n$ & $M(D E)$ & $95 \% / C$ & $n$ & $M(D E)$ & IC $95 \%$ & \\
\hline \multicolumn{8}{|l|}{ Expresiones violentas } \\
\hline Entre cónyuges & 568 & $0.21(.33)$ & {$[-.25,-.08]$} & 84 & $0.38(.48)$ & {$[-.27,-.05]$} & 0.42 \\
\hline De padres a hijos & 565 & $0.28(.35)$ & {$[-.33,-.15]$} & 85 & $0.53(.56)$ & {$[-.37,-.12]$} & 0.55 \\
\hline De hijos a padres & 560 & $0.17(.24)$ & {$[-.21,-.09]$} & 84 & $0.32(.33)$ & {$[-.22,-.07]$} & 0.53 \\
\hline Entre hermanos & 554 & $0.46(.47)$ & {$[-.37,-.15]$} & 85 & $0.72(.51)$ & {$[-.38,-.14]$} & 0.53 \\
\hline \multicolumn{8}{|l|}{ Tipo de violencia } \\
\hline Física & 560 & $0.52(.48)$ & {$[-.39,-.16]$} & 83 & $0.80(.55)$ & {$[-.40,-.14]$} & 0.37 \\
\hline Psicológica & 557 & $0.22(.30)$ & {$[-.31,-.16]$} & 83 & $0.47(.45)$ & {$[-.34,-.14]$} & 0.48 \\
\hline Sexual & 565 & $0.01(.07)$ & {$[-.03, .00]$} & 87 & $0.03(.09)$ & {$[-.03, .00]$} & 0.17 \\
\hline
\end{tabular}

Nota: $n=$ número de sujetos; $M=$ calificación promedio; $D E=$ desviación estándar; $I C=$ intervalo de confianza

En ambos grupos predominó la violencia física, seguida por la de tipo psicológico y en tercer lugar la de tipo sexual. Los Usuarios de drogas ilícitas reportaron una calificación promedio significativamente mayor en violencia física, $t$ de Student $(101.262)=4.294, p=.000$, y psicológica en el contexto familiar, $t$ de Student (92.874) $=4.701, p=.000)$. En este mismo grupo también se reportó una mayor violencia sexual, aunque no fue significativa (Tabla 3 ).

El tamaño del efecto obtenido en la violencia física y psicológica fue mediano (entre .20 y .50), si bien fue bajo en la violencia sexual (menor a .20).

\section{Predictores del consumo de drogas ilícitas alguna vez}

Como parte de los objetivos del estudio, una vez identificado el consumo de sustancias y de explorar diferencias con respecto a las distintas áreas de la violencia familiar entre los No usuarios con los Usuarios de drogas ilícitas, se procedió a identificar qué características pudiesen fungir como predictoras. Previo al análisis se correlacionaron las variables incluidas en el modelo, encontrando valores moderados (entre 21 y .80 ), lo cual permite suponer cierta colinealidad. Los resultados de la regresión logística mostraron que, en la población estudiada, la

Tabla 4

Variables del modelo para identificar predictores del consumo de drogas ilícitas $(N=667)$

\begin{tabular}{|c|c|c|c|}
\hline Variable independiente & $B$ & $\operatorname{Exp}(B)$ & IC 95\% \\
\hline violencia entre cónyuges & 0.383 & 1.466 & {$[0.76,2.79]$} \\
\hline violencia de padres a hijos & 0.135 & 1.145 & {$[0.43,2.98]$} \\
\hline violencia de hijos a padres & 0.552 & 1.737 & {$[0.43,6.98]$} \\
\hline violencia entre hermanos & $0.657^{*}$ & 1.929 & {$[1.09,3.41]$} \\
\hline
\end{tabular}

Nota: $N=$ número de participantes, ${ }^{*} p<.05$ 
violencia familiar entre hermanos funge como un predictor del consumo de drogas lícitas (Tabla 4).

\section{CONCLUSIONES}

En general, el estudio muestra que los estudiantes que participaron perciben con poca frecuencia la presencia de situaciones relacionadas con la violencia en su grupo familiar; sin embargo, quienes señalan haber vivido algún evento de esta índole probablemente estén en una condición de vulnerabilidad emocional que puede devenir en el uso de alguna sustancia psicoactiva, como una forma de atenuar ese malestar afectivo. Estos hallazgos coinciden con lo reportado en otros estudios realizados en población adolescente (Suárez \& Menkes, 2006; Velasco Fernández, 2009).

Asimismo, los resultados muestran una participación ligeramente mayor de mujeres; y al comparar los grupos de No usuarios con los Usuarios de drogas ilícitas, se observó que se mantuvo una mayor participación de mujeres en el grupo de usuarios de drogas, contrario a lo esperado, según lo reportado en otras encuestas sobre consumo de drogas que muestran todavía un mayor consumo en los varones (Instituto Nacional de Psiquiatría Ramón de la Fuente Muñiz, Comisión Nacional Contra las Adicciones, Secretaría de Salud, 2015; Secretaría de Salud, Instituto Nacional de Psiquiatría Ramón de la Fuente Muñiz, Secretaría de Educación Pública, 2016).

Llama la atención que un porcentaje importante de estudiantes que conformaron el grupo de usuarios de drogas se encontraba estudiando el tercer año de secundaria, lo que permite fortalecer la idea de que conforme avanza la edad hay un mayor número de jóvenes que pueden involucrarse en el uso de sustancias psicoactivas (Herrera-Vázquez, Wagner, Velasco-Mondragón, Borges, \& Lazcano-Ponce, 2004).

Por otra parte, también se identificó que un mayor porcentaje de alumnos que han usado alguna droga, además de estudiar, trabajan, lo que permite complementar el gasto familiar pero también implica un riesgo para el consumo de sustancias psicoactivas, debido a que cuentan con cierto poder adquisitivo (Arellanez Hernández, Diaz Negrete, Wagner Echeagaray, \& Pérez Islas, 2004).

Particularmente en la exploración acerca de la violencia en el contexto familiar, como se ha señalado, se percibe con poca frecuencia, pero hay diferencias sustantivas entre los no usuarios y usuarios de drogas, pues en estos últimos hay una mayor ocurrencia de eventos con violencia entre hermanos, seguida por actos violentos de los padres hacia los hijos, de éstos hacia los padres y entre cónyuges. Estos hallazgos coinciden con lo reportado en un estudio en población adolescente del estado de Veracruz (Tlaxcalteco González, Arellanez Hernández, \& Márquez Barradas, 2017).

Particularmente llama la atención que sí se ha registrado la presencia de violencia de los hijos hacia los padres, elemento que se ha venido evaluando en adolescentes españoles (Calvete, Gámez-Guadix, \& Orue, 2014; Calvete et al., 2011), y que hace evidente la necesidad de seguir profundizando en las consecuencias que puede traer aparejada la violencia filio-parental con el consumo de sustancias psicoactivas.

Asimismo, destaca que los jóvenes que han usado drogas con mayor frecuencia reportan violencia física y psicológica en su familia. Un elemento que en sí mismo es relevante es que, aunque sólo algunos casos reportaron violencia sexual (golpear los órganos sexuales, manipular alguna situación para propiciar actos sexuales y/o presionar para tener relaciones sexuales), ésta sí existe en el grupo familiar o por lo menos algunos alumnos así lo señalaron. Esto coincide con los hallazgos reportados por la ENDIREH realizada en el año 2016 (INEGI, 2017).

Al explorar los elementos predictores del consumo de drogas, se encontró que a mayores condiciones de violencia en el grupo familiar, hay una mayor probabilidad de que los estudiantes que viven situaciones de violencia entre hermanos puedan estar en mayor riesgo de consumir algún tipo de sustancias ilícitas. Este dato es relevante, pues al parecer no se ha puesto atención a la forma en la que se relacionan los hermanos y las repercusiones que puede haber en quienes sufren de violencia por parte de ellos.

Si bien los hallazgos obtenidos no son generalizables, sí ofrecen elementos de que los estudiantes participantes en el estudio perciben una frecuencia relativamente baja de situaciones de violencia familiar, aunque como se señaló en otro momento eso no quiere decir que no exista, quizás se está naturalizando, se está convirtiendo en algo que forma parte de lo cotidiano, ya que como lo señaló León Carmona (2016), al menos en el municipio de Alto Lucero se ha incrementado la presencia de eventos violentos a tal grado que está dejando de ser preocupante que día a día se presenten extorsiones, robos, asaltos y levantones. Valdría la pena en estudios posteriores continuar profundizando en este riesgo que puede estar latente (la naturalización de la violencia), así como incorporar un diseño muestral que permita generalizar los hallazgos obtenidos.

Finalmente, los resultados obtenidos sugieren por un lado la necesidad de construir o diseñar actividades que permitan el desarrollo de habilidades sociales centradas en la familia, particularmente para atenuar las manifestaciones de violencia en la convivencia entre hermanos, así como el manejo de emociones; por otro lado, sugieren la necesidad de profundizar en investigaciones que 
manejen las mismas variables, permitan corroborar los hallazgos encontrados para favorecer la atención de la salud mental de los jóvenes, la construcción y el desarrollo de políticas públicas en el contexto familiar.

\section{FUENTE DE FINANCIAMIENTO}

El presente manuscrito forma parte de un proyecto más amplio que fue financiado por la Secretaría de Educación Pública, a través de la Convocatoria de PRODEP, de Apoyo a la Incorporación de Nuevos PTC, con número de oficio DSA/103.5/16/10438. Cabe señalar que, como parte de la perspectiva del proyecto, las opiniones, los hallazgos y las conclusiones que son presentadas en el manuscrito son de los autores y no necesariamente reflejan opiniones o puntos de vista del patrocinador.

\section{CONFLICTOS DE INTERÉS}

Los autores del manuscrito declaramos que no existen conflictos de interés en cuanto a los datos presentados.

\section{AGRADECIMIENTOS}

Los autores del manuscrito agradecen el apoyo de la Mtra. Rosa Natalia Rodríguez Álvarez y María Antonia Pérez Sosa, representantes de la Zona de Supervisión Xalapa "A", para la realización del proyecto de investigación. A las autoridades y orientadores de los Telebachilleratos Alto Lucero, Blanca espuma, Chiltoyac, Cocoatzintla, Colonia Guadalupe Victoria (223E), Colonia Guadalupe Victoria (804K), Colonia Higueras, Colonia Revolución, El cafetal, El Castillo, La Reforma, La Unión, Mesa de Guadalupe, Reserva Territorial Tronconal y Trapiche del Rosario, que al participar en la capacitación del proyecto y en el levantamiento de información, lo hicieron posible. Finalmente, los autores agradecen ampliamente a cada uno de los estudiantes que contestaron el instrumento, por compartir sus opiniones y puntos de vista de los temas evaluados.

\section{REFERENCIAS}

Arellanez Hernández, J. L., Diaz Negrete, D. B., Wagner Echeagaray, F., \& Pérez Islas, V. (2004). Factores psicosociales asociados con el abuso y la dependencia de drogas entre adolescentes: Análisis bivariados de un estudio de casos y controles. Salud Mental, 27(3), 54-64.

Asociación Médica Mundial (2013). Declaración de Helsinki. Principios éticos para las investigaciones médicas en seres humanos (64a Asamblea General, Fortaleza, Brasil). Recuperado de http://www. isciii.es/ISCIII/es/contenidos/fd-investigacion/fd-evaluacion/fdevaluacion-etica-investigacion/Declaracion-Helsinki-2013-Esp.pdf
Becoña, E., Martínez, Ú., Calafat, A., Juan, M., Duch, M., \& Fernández-Hermida, J. R. (2012). ¿Cómo influye la desorganización familiar en el consumo de drogas de los hijos? Una revisión. Adicciones, 24(3), 253-268. doi:10.20882/adicciones.97

Butters J. (2002). Family stressors and adolescent cannabis use: a pathway to problem use. Journal of Adolescence, 25(6), 645654. doi:10.1006/jado.2002.0514

Caballero, M. A., \& Ramos, L. (2004). Violencia: una revisión del tema dentro del marco de trabajo de investigación en el Instituto Nacional de Psiquiatría. Salud Mental, 27(2), 21-30.

Cabrera-Fuentes, D. M. (2011). Estudio de la correlación múltiple entre el bullying, funcionalidad familiar y bajo rendimiento escolar cognitivo en las asignaturas de lenguaje y matemática. (Tesis de licenciatura inédita). Universidad Central del Ecuador, Facultad de Ciencias Psicológicas.

Calvete, E., Orue, I., \& Sampedro, R. (2011). Violencia filio-parental en la adolescencia: Características ambientales y personales. Infancia y Aprendizaje / Journal for the Study of Education and Development, 34(3), 349-363. doi:10.1174/021037011797238577

Calvete, E., Gámez-Guadix, M., \& Orue, I. (2014). Características familiares asociadas a violencia filio-parental en adolescentes. Anales de Psicología, 30(3), 1176-1182. doi:10.6018/analesps.30.3.166291

Centros de Integración Juvenil (2007). Violencia familiar y adicciones. Recomendaciones preventivas. México: ClJ.

Corsi, J. (1994). Una mirada abarcativa sobre el problema de la violencia familiar. En J. Corsi, (Comp.), Violencia familiar, Una mirada interdisciplinar sobre un grave problema social (pp. 1563). México: Paidós

Diario Oficial de la Federación. (2014). Reglamento de la Ley General de Salud en Materia de Investigación para la Salud. Última Reforma DOF 02-04-2014. México. Recuperado de https://www. uv.mx/ics/files/2016/05/Reg_LGS_MIS.pdf

Diario Oficial de la Federación. (2017). Ley General de Salud. Última Reforma DOF 08-12-2017. México. Recuperado de http://www. diputados.gob.mx/LeyesBiblio/pdf/142_081217.pdf

Dirección General de Telebachillerato (2017). ¿Quiénes somos? Recuperado de: http://www.sev.gob.mx/tebaev/category/direccion/

El-Sheikh, M., \& Flanagan, E. (2001). Parental problem drinking and children's adjustment: Family conflict and parental depression as mediator and moderators of risk. Journal of Abnormal Child Psychology, 29(5), 417-32. doi: 10.1023/A:1010447503252

Gallegos-Guajardo, J., Ruvalcaba-Romero, N. A., Castillo-López, J., \& Ayala-Díaz, P. C. (2016). Funcionamiento familiar y su relación con la exposición a la violencia en adolescentes mexicanos. Acción Psicológica, 13(2), 69-78. doi: 10.5944/ap.13.2.17810

Harrington, C., \& Metzler, A. (1997). Are adult children of dysfunctional families with alcoholism different from adult children of dysfunctional families without alcoholism? A look at committed, intimate relationships. Journal of Counseling Psychology, 44(1),102-107. doi:10.1037/0022-0167.44.1.102

Herrera-Vázquez, M. Wagner, F. A., Velasco-Mondragón, E., Borges, G., \& Lazcano-Ponce, E. (2004). Inicio en el consumo de alcohol 
y tabaco y transición a otras drogas en estudiantes de Morelos, México. Revista Salud Pública de México, 46(2), 132-140.

Instituto Nacional de Estadística y Geografía (2003). Violencia intrafamiliar. Encuesta 1999. Documento metodológico y resultados. México, INEGI.

Instituto Nacional de Estadística y Geografía (2017). Encuesta Nacional sobre la Dinámica de las Relaciones en los Hogares (ENDIREH) 2016. Boletín de prensa núm. 379/17. Recuperado de: http://www.inegi.org.mx/saladeprensa/boletines/2017/endireh/ endireh2017_08.pdf

Instituto Nacional de Psiquiatría Ramón de la Fuente Muñiz, Comisión Nacional Contra las Adicciones, Secretaría de Salud (2015). Encuesta Nacional de Consumo de Drogas en Estudiantes 2014: Reporte de Drogas. México: INPRFM. Recuperado de: www.inprf.gob.mx, www.conadic.gob.mx, www.cenadic.salud. gob.mx

Krug, E. G., Dahlberg, L. L., Mercy, J. A., Zwi, A. B., \& Lozano, R. (2003). Capítulo 1. La violencia, un problema mundial de salud pública. En Oficina Regional para las Américas de la Organización Mundial de la Salud (Ed.), El Informe mundial sobre la violencia y la salud (pp. 1-24). Washington: OPS, Oficina Regional para las Américas de la Organización Mundial de la Salud. Recuperado de: http://apps.who.int/iris/bitstream/10665/112670/1/9275315884_ spa.pdf

Ledoux, S., Miller, P., Choquet, M., \& Plant, M. (2002). Family structure, parent child relationships, and alcohol and other drug use among teenagers in France and the United Kingdom. Alcohol \& Alcoholism, 37(1), 52-60. doi:10.1093/alcalc/37.1.52

León Carmona, M. A. (2016). La gente está huyendo de Alto Lucero. PosData, noticias de Veracruz [Noticias en un blog]. Recuperado de: http://www.blog.expediente.mx/nota/20941/periodico-deveracruz-portal-de-noticias-veracruz/la-gente-esta-huyendode-alto-lucero
Martínez Almodóvar, M. B., López Triana, A. E., Díaz Montesinos, A., \& Teseiro Plá, M. M. (2015). Violencia intrafamiliar y trastornos psicológicos en niños y adolescentes del área de salud de Versalles, Matanzas. Revista Médica Electrónica, 37(3), 237-245. Recuperado de http://scielo.sld.cu/scielo.php?script=sci_ arttext\&pid=S1684-18242015000300006\&lng=es\&tIng=es.

Secretaría de Salud (2009). Norma Oficial Mexicana NOM-046-SSA22005, Violencia familiar, sexual y contra las mujeres. Criterios para la prevención y atención. México: Diario Oficial de la Federación. Secretaría de Salud, Instituto Nacional de Psiquiatría Ramón De La Fuente Muñiz, Instituto Nacional de Salud Pública, Comisión Nacional contra las Adicciones (2016). Encuesta Nacional de consumo de drogas, alcohol y tabaco 2016-2017. Consumo de drogas: prevalencias globales, tendencias y variaciones estatales. México: SSA. Recuperado de: https://www.gob.mx/cms/uploads/ attachment/file/234856/CONSUMO_DE_DROGAS.pdf

Suárez, L., \& Menkes, C. (2006). Violencia familiar ejercida en contra de los adolescentes mexicanos. Revista de Saúde Pública, 4O(4), 611-619. doi:10.1590/s0034-89102006000500009

Tlaxcalteco-González, A., Arellanez-Hernández, J. L., \& Márquez-Barradas, M. L. (abril, 2017). Validación de Escala sobre Percepción de la Violencia Familiar en Adolescentes Mexicanos. En M. X. Mercado-Rivas, M. L. Sánchez-Aranda, \& M. C. Pérez-Pimienta (Comp.), Psicología integral al servicio de la humanidad, XLIV Congreso Nacional del Consejo Nacional para la Enseñanza e Investigación en Psicología A.C. - Universidad Autónoma de Nayarit (pp. 162-183).

Uribe, A. F., Orcasita, L., \& Aguillón, E. (2012). Bullying, redes de apoyo y funcionamiento familiar en adolescentes de una institución educativa de Santander, Colombia. Psychologia, Avances de la Disciplina, 6(2), 83-99.

Velasco Fernández, R. (2009). Psicopatología de la edad juvenil: Temas Seleccionados. México: Universidad Veracruzana. 